\title{
LA INMIGRACIÓN VENEZOLANA EN EL PERÚ: DESAFÍOS Y OPORTUNIDADES DESDE LA PERSPECTIVA DE LA SALUD
}

\author{
Walter Mendoza ${ }^{1, a}$, J. Jaime Miranda²,b
}

\begin{abstract}
RESUMEN
Resultado de la crisis política, social y económica en la República Bolivariana de Venezuela, más de setecientas mil personas han inmigrado al Perú desde el segundo semestre del 2017. En el año posterior a los censos del 2017, la población en el Perú creció en cerca de un millón de habitantes, unos trescientos mil de ellos peruanos, el resto inmigrantes venezolanos, predominantemente jóvenes. En este artículo se describe y analiza la situación e implicancias para la salud del hecho que Perú se convirtiera en país de destino migratorio. Para ello, se hace el análisis secundario de encuestas aplicadas a la población venezolana en el Perú. Los desafíos principales derivan del limitado acceso a la atención en salud. La atención en salud sexual y reproductiva muestra los mayores déficits, por debajo de las poblaciones urbanas del Perú. Las vulnerabilidades de la población migrante venezolana no están al margen de las que ya afrontaban las poblaciones urbanas más pobres del Perú, cuyos servicios tampoco satisfacen sus necesidades y demandas, ni en cobertura ni en calidad. No obstante, la inmigración también genera oportunidades, como la que representan los profesionales y técnicos de la salud, que pudieran contribuir a compensar el déficit generado por la emigración de miles de profesionales de la salud peruanos/as en las últimas décadas. Oportunidad también para no perder de vista que las desigualdades en el derecho a la salud siguen siendo retos para avanzar hacia un desarrollo inclusivo.
\end{abstract}

Palabras clave: Emigración internacional; Encuesta; Inmigración internacional; Perú; Venezuela. (Fuente: DeCS BIREME).

\section{VENEZUELAN IMMIGRATION IN PERU: CHALLENGES AND OPPORTUNITIES FROM A HEALTH PERSPECTIVE}

\begin{abstract}
As a result of the political, social, and economic crisis in the Bolivarian Republic of Venezuela, more than 700,000 people have immigrated to Peru since the second semester of 2017. In the year following the 2017 census, Peru's population grew by nearly one million, some 300,000 of them Peruvian, the rest being predominantly young Venezuelan immigrants. This article describes and analyzes the situation and health implications stemming from the fact that Peru became a migratory destination. To this end, a secondary analysis of surveys applied to the Venezuelan population in Peru was carried out. The main challenges arise from limited access to healthcare. Sexual and reproductive healthcare shows the largest deficits, below Peru's urban populations. The vulnerabilities of the Venezuelan migrant population are not detached from those already faced by Peru's poorest urban populations, whose services do not meet their needs and demands, neither in terms of coverage nor quality. However, immigration also generates opportunities, such as that represented by health professionals and technicians, who could contribute to offset the deficit generated by the emigration of thousands of Peruvian health professionals in recent decades. It is also an opportunity not to lose sight of the fact that inequalities in the right to healthcare are still challenges to inclusive development.
\end{abstract}

Keywords: International emigration, International immigration, Peru, Survey, Venezuela (Source: MeSH NLM).

\footnotetext{
Fondo de Población de las Naciones Unidas (UNFPA). Lima, Perú

CRONICAS, Centro de Excelencia en Enfermedades Crónicas, Universidad Peruana Cayetano Heredia. Lima, Perú,

Médico cirujano; ${ }^{\mathrm{b}}$ Médico, magíster y doctor en Epidemiología

Recibido: 08/08/2019 Aprobado: 04/09/2019 En línea: 23/09/2019
}

Citar como: Mendoza W, Miranda JJ. La inmigración venezolana en el Perú: desafíos y oportunidades desde la perspectiva de la salud. Rev Peru Med Exp Salud Publica. 2019;36(3):497-503. doi: http://dx.doi.org/10.17843/rpmesp.2019.363.4729. 


\section{INTRODUCCIÓN}

Hablar de migración en el Perú solía referirse a la que venía del campo hacia las ciudades. En los últimos años la discusión pública empezó a derivar hacia la emigración. Buena parte de ella partió en los años 70 y 80 hacia Estados Unidos y países de América Latina, principalmente Venezuela, y desde los 90 a Argentina y Chile. Desde 1990 han emigrado unos tres millones de peruanos/as ${ }^{(1)}$.

En la última década la situación empezó a cambiar, iniciándose inclusive una migración de retorno. Lo que no estaba previsto era convertirnos en país de destino migratorio, esta vez desde la República Bolivariana de Venezuela. Según las Naciones Unidas han emigrado aproximadamente cuatro millones de personas, de los que hacia mediados del 2019 casi una quinta parte llegaron al Perú (2). Además de las ventajas ofrecidas por el gobierno peruano, entre las explicaciones se debe considerar las relaciones de parentesco y relaciones sociales establecidas por quienes emigraron hacia Venezuela en los 70 y 80.

Un informe reciente de la Alta Comisionada de las Naciones Unidas para los Derechos Humanos, a cargo de Michelle Bachelet, destaca que «en cuanto al derecho a la salud en Venezuela, la situación es grave» (deterioro en la infraestructura y equipos, éxodo de personal técnico y profesional, reaparición de enfermedades evitables con vacunas, escasez de anticonceptivos, ausencia de información, etc.) ${ }^{(3)}$. Situación que en parte explica la experiencia migratoria, la cual a la luz de los Objetivos del Desarrollo Sostenible (ODS) ha adquirido mayor visibilidad. El foco que antes estaba en otras regiones del mundo, ya incluye a nuestra región y a nuestro país.

Desde la perspectiva del derecho a la salud, ¿Cuáles son las principales necesidades y demandas de la población venezolana llegada al Perú en los últimos dos años? y ¿Qué sabemos y qué necesitamos saber acerca de esta población? Finalmente, ¿Qué beneficios potenciales trae consigo esta migración, por ejemplo, con sus profesionales y técnicos de la salud, y qué nuevos retos plantean? En este artículo también exploramos la necesidad de generar nueva información, sea mediante investigaciones o a partir de la información que ya generan las encuestas de base poblacional o los registros administrativos.

\section{¿QUÉ SABEMOS DE LA POBLACIÓN VENEZOLANA EN EL PERÚ?}

El deterioro de las condiciones de vida de la población venezolana, incluyendo la falta de servicios y desabastecimiento de medicamentos, suministros y equipos de salud, habrían determinado su inopinada emigración ${ }^{(4)}$.
Los estados tienen la obligación de proteger el derecho a la salud en el marco de los tratados internacionales que han suscrito, al no contarse con aseguramiento universal tal derecho no se ejerce cabalmente. Como es el caso del Perú, en el que, a pesar de los avances registrados en los últimos años, persisten importantes brechas en cobertura y calidad ${ }^{(5)}$.

Una primera imagen de la población venezolana en el Perú, la cual rápidamente devino desactualizada, provino de los resultados de los Censos del 22 de octubre del 2017. A esa fecha se contaron 60949 personas, que habían llegado del 2012 al 2017, y aun antes; vale decir, respectivamente, como migrantes recientes y como migrantes de toda la vida, según muestra el siguiente cuadro ${ }^{(6)}$ :

La imagen del 2017 mostraba rasgos que, más allá del volumen poblacional, al momento de realizar la Encuesta sobre las Condiciones de Vida de la Población Venezolana que reside en Perú (ENPOVE, noviembre y diciembre del 2018), no se habían modificado sustancialmente: la mayor parte seguía en los principales centros urbanos (86\% en Lima y Callao al 2017). Un año después, se estimaba en 630715 , de la que 568570 estaban en la capital, y 40370 en el Callao, en conjunto $96,5 \%$ del total en el país ${ }^{(7)}$.

\section{FUENTES Y MÉTODOS}

Para este artículo nos basamos en dos fuentes, una principal es la ENPOVE, aplicada en Arequipa, Callao, Cusco, Lima, Trujillo y Tumbes, en una muestra de 3611 viviendas. De ella, hacemos uso de algunos módulos de interés directo para la salud, incluyendo los de discriminación y violencia, educación, y opiniones sobre relaciones de género. Los diagnósticos son auto-reportados.

Otro aspecto de interés explorado por esta encuesta es el perfil educativo de la población venezolana llegada recientemente al Perú, la cual incluye miles de profesionales y técnicos de salud.

En menor medida, también hacemos uso del Monitoreo de Flujo de la Población Venezolana en el Perú, administrada por la Organización Internacional para las Migraciones (OIM) y UNICEF ${ }^{(8)}$. Con distinta representatividad estadística a la ENPOVE, se aplica periódicamente en los Centros Binacionales de Atención Fronteriza (CEBAF) de Tacna y Tumbes, sólo en una ocasión (julio 2018) fue aplicada también en áreas de mayor afluencia de población venezolana en Lima. Adicionalmente, en cuanto fuera pertinente, y a modo de referencia, se comparan dichos resultados con otras similares aplicadas para la población urbana del Perú, aunque no necesariamente compartan las mismas metodologías (Tabla 1). 
Tabla 1. Población censada en Perú procedente de Venezuela, 2017

\begin{tabular}{|c|c|c|c|}
\hline Población procedente de Venezuela & Total & Hombres & Mujeres \\
\hline Total & 60949 & 32957 & 27992 \\
\hline Nacidos en Venezuela & 47481 & 25502 & 21979 \\
\hline Llegaron entre $2012-2017$ & 38465 & 20986 & 17479 \\
\hline Llegaron antes de 2012 & 9016 & 4516 & 4500 \\
\hline Peruanos que llegaron de Venezuela entre 2012-2017 & 12870 & 7146 & 5724 \\
\hline $\begin{array}{l}\text { Personas de otras nacionalidades que llegaron de } \\
\text { Venezuela entre } 2012-2017\end{array}$ & 598 & 309 & 289 \\
\hline
\end{tabular}

Fuente: INEI. Censo Nacional XII de Población y VII de Vivienda, 2017

\section{ASPECTOS SOCIO DEMOGRÁFICOS}

Un primer rasgo de interés para la salud derivado de la ENPOVE es la estructura de edades. Según la tabla 2, que compara en términos porcentuales edades del ciclo de vida en migrantes venezolanos y la proyectada para el Perú del 2018 (que ya los incluye), el grupo de 18 a 44 años representa cerca de tres cuartas partes de dicha población, mientras que en la del Perú bordea el $40 \%$. A su vez, la población adulta mayor representa el 1,6\%, mientras que la del Perú bordea el $12 \%$. La población en la primera infancia (0 a 5 años) representa el 7,9\%, mientras que en el Perú es el $10,5 \%{ }^{(9)}$.

Entre febrero y octubre 2018, esta inmigración representaría poco más de $2 \%$, que se agrega al $1 \%$ anual en los últimos años. Como cabe esperar, su perfil epidemiológico, necesidades y demandas, interacciones sociales, así como los condicionantes de su vulnerabilidad (la desprotección ante daños potenciales, sean físicos, sociales, económicos o legales) ${ }^{(10)}$, están influenciados por el perfil de edades.

\section{HALLAZGOS SOBRE SITUACIÓN, NECESIDADES Y DEMANDAS EN SALUD}

Según la ENPOVE, el $11 \%$ de la población inmigrante venezolana refirió tener algún problema de salud crónico (no verificada mediante examen), $12,9 \%$ en mujeres, $9,3 \%$ en hombres. Para el mismo periodo de realización de la encuesta, para la población urbana del Perú este valor era $39 \%{ }^{(11)}$, probablemente influenciado por la distinta estructura de edades, más joven en la población venezolana. Según advertimos estas comparaciones deben ser hechas con cautela.

Dentro del $11 \%$ de quienes reportaron enfermedad crónica, las más comunes fueron asma (39\%), seguido de hipertensión (18,4\%), artritis $(4,8 \%)$ y diabetes con $4,6 \%$. El cáncer también fue reportado en $2,1 \%$ de entrevistados/as con alguna enfermedad crónica, $3,4 \%$ en mujeres y $0,5 \%$ en hombres.

En cuanto al tratamiento, ocho de cada diez de quienes reportaron alguna enfermedad crónica manifestó no recibirlo. Sólo el $11,5 \%$ de quienes lo requieren reciben el tratamiento con la frecuencia necesaria a su condición, $13,4 \%$ en mujeres y $9,1 \%$ en hombres. Para la población urbana del Perú con alguna enfermedad crónica, seis de diez no buscaron atención.

Asimismo, la ENPOVE indagó acerca de algún padecimiento (accidentes, síntoma, malestar o enfermedad) presentado desde que llegaran al Perú, 44\% presentó algún problema de salud, la mitad de naturaleza respiratoria o gastrointestinal. Para el mismo periodo de dicha encuesta,

Tabla 2. Estructuras de edades de la población venezolana en Perú y de la población del Perú, 2018

\begin{tabular}{|c|c|c|c|c|c|c|}
\hline \multirow{2}{*}{ Ciclo de vida (años) } & \multicolumn{3}{|c|}{ Población venezolana en Perú (\%) } & \multicolumn{3}{|c|}{ Población del Perú (\%) } \\
\hline & Total & Hombres & Mujeres & Total & Hombres & Mujeres \\
\hline Primera infancia (0 a 5) & 7,9 & 7,8 & 8,1 & 10,5 & 10,8 & 10,2 \\
\hline Niñez (6 a 11) & 6,3 & 6,1 & 6,5 & 12,1 & 12,4 & 11,9 \\
\hline Adolescencia (12 a 17) & 4,5 & 4,6 & 4,4 & 10,0 & 10,1 & 9,8 \\
\hline Jóvenes (18 a 29) & 42,0 & 41,7 & 42,3 & 20,0 & 19,8 & 20,1 \\
\hline Adultos/as jóvenes (30 a 44) & 29,8 & 32,3 & 29,7 & 21,7 & 22,0 & 21,5 \\
\hline Adulta/o (45 a 59) & 8,0 & 6,5 & 9,7 & 15,4 & 15,2 & 15,7 \\
\hline Adultas/os mayores (60 a más) & 1,6 & 1,1 & 2,2 & 12,0 & 11,5 & 12,5 \\
\hline
\end{tabular}

Fuente: INEI. ENPOVE, 2019.

INEI. Perú: Estimaciones y Proyecciones de la Población Nacional, por Años Calendario y Edades Simples, 1950-2050, 2019. 
y referida a las cuatro semanas anteriores, $30 \%$ de la población urbana en el Perú reportaba algún problema de salud no crónico.

Entre quienes buscaron atención, el 55,1\% lo hizo en una botica o farmacia, mientras que un tercio se automedicó, y poco menos del $20 \%$ acudió a un establecimiento del MINSA. Aunque no estrictamente comparables en el periodo de referencia, la población urbana del Perú que buscó atención fue $48 \%{ }^{(11)}$. De estos, aproximadamente la cuarta parte acudió a algún establecimiento del MINSA, uno de cada tres se automedicó, mientras que dos de cada cinco fueron a una farmacia. Esta opción es la que más ha crecido entre quienes buscan atención, pasando de 10 a $20,5 \%$ en las áreas urbanas ${ }^{1}$, del 2004 al $2018^{(12)}$.

Otro aspecto importante fue la condición de discapacidad. Acaso por no tratarse de una encuesta especializada, en una breve sección la ENPOVE engloba sus distintos tipos (definidas como la «dificultad física, mental, intelectual o de los sentidos de manera permanente»). Sin especificarlos, se encontró que $2 \%$ refería algún tipo de discapacidad, por debajo de la encuesta especializada realizada en el país el $2012(5,2 \%)$. No se presentan resultados sobre su acceso a tratamiento.

Respecto al aseguramiento, al momento de la realización de la ENPOVE sólo el 8,5\% refirió contar con un seguro de salud, la novena parte de la población peruana. Poco más de la mitad de quienes cuentan con algún seguro está afiliado al Seguro Integral de Salud (SIS).

\section{SALUD SEXUAL Y REPRODUCTIVA}

Las mujeres en edad reproductiva, de 15 a 49 años, representan aproximadamente a tres cuartas partes de las venezolanas en el Perú. Tres de cada diez mayores de quince años fueron atendidas en servicios de salud sexual o reproductiva, mientras que en caso de los hombres uno de cada cuatro.

El uso de métodos modernos de planificación familiar en mujeres en edad fértil unidas, era sólo el $20,6 \%$, muy por debajo del promedio de peruanas urbanas $(56,4 \%)$, que a su vez está por debajo del promedio regional, en torno al $70 \%$. Contrastando estos resultados con los embarazos en venezolanas de 12 a 49 años al momento de la encuesta (4\%, sin incluir al $1,6 \%$ que no lo sabe o no está segura), y los bajos niveles de uso de métodos modernos, lo más probable es que no fueron planificados.

En cuanto al VIH/SIDA, 0,7\% de mujeres y $0 \%$ de hombres reportaron tenerlo. No obstante, probablemente se trata de una subestimación no solo porque el diseño de la muestra estadística no estuvo orientado a identificar a la población con dicho diagnóstico, ni hubo confirmación más allá de la proporcionada por la persona encuestada, al margen de consideraciones de confidencialidad. Por su parte, otras fuentes señalan que el desabastecimiento de tratamiento en la República Bolivariana de Venezuela explicaría en parte la emigración de la población seropositiva ${ }^{(13)}$.

Según un estudio reciente orientado a describir la situación y generar recomendaciones para atender las necesidades y demandas de la población venezolana con VIH/Sida ${ }^{(14)}$, hacia fines del 2018, se contaban 1338 personas recibiendo el TARGA, del $3 \%$ al $4 \%$ del total nacional. Las redes de solidaridad y organizaciones de la sociedad civil fueron las primeras en movilizarse para este fin. $Y$ si bien son conocidas las barreras para la población peruana que comparte características socio-demográficas similares a la migrante con $\mathrm{VIH} / \mathrm{Sida}$, en su caso se deben agregar otras barreras, p.e. para recibir al pre-TARGA, gastos de bolsillo y el registro en el SIS. Según reportaron organizaciones de la sociedad civil, hacia fines del 2018 se habrían producido dieciséis muertes de inmigrantes venezolanos con $\mathrm{VIH} /$ Sida.

\section{EXPERIENCIAS DE DISCRIMINACIÓN Y VIOLENCIA}

También se indagó acerca de experiencias de discriminación, esta fue reportada por uno de cada tres venezolanos, dos terceras partes en la calle o en lugares públicos, muy pocos en algún establecimiento de salud (3,4\%, inferior al promedio para la población peruana).

Según la ENPOVE el maltrato físico en quienes tenían 18 y más años, fue de uno de cada diez entrevistados. La mitad de perpetradores fueron extraños, la tercera parte el esposo/a o compañero/a. Sólo tres de cada diez solicitaron ayuda, principalmente en comisarías (tres de cada cuatro casos). La cuarta parte no acudió a ninguna institución porque no sabía a dónde hacerlo.

El acoso en sus distintas modalidades (sexual, laboral, escolar o ciberacoso) también fue reportado por el 17,8\% de mujeres venezolanas, principalmente en adolescentes de 12 a 17 años (una de cada cinco). A su vez, 2,9\% refirió conocer a alguna víctima de violencia sexual (sin identificar al agresor). Según la ENDES 2018, en el caso de las mujeres de 15 a 49 años unidas en áreas urbanas del Perú la violencia sexual por parte de sus parejas fue $2,5 \%$ en los últimos doce meses. Cerca de dos terceras partes percibe que en Perú la violencia sexual es mayor en su país de origen, lo mismo que la violencia física.

\section{POBLACIÓN VENEZOLANA CON ESTUDIOS EN CARRERAS DE SALUD}

Desde la perspectiva de la salud poblacional, sería insuficiente caracterizar la reciente inmigración venezolana únicamente como una irrupción inesperada de necesidades 
y demandas de atención. La ENPOVE también incluyó un módulo de estudios y empleo, identificándose técnicos y profesionales de la salud o estudiantes con estudios incompletos. Hacia fines del 2018 la cuarta parte tenía estudios superiores completos, uno de cada cinco hombres, tres de cada diez mujeres. Uno de cada siete tenía estudios superiores incompletos. En el caso de las carreras técnicas, uno de cada cinco las había estudiado, uno de cada siete las había terminado.

El 92,2\% del total de profesionales refirió haber obtenido el título. No obstante, la mayoría no ha podido homologarlos, principalmente por razones económicas (cuatro de cada diez), por no conocer el procedimiento (la tercera parte), o porque no trajeron sus diplomas (uno de cada cinco).

Entre quienes tienen estudios profesionales, completos o incompletos, el 3,6\% estudió enfermería, 2,4\% medicina, $1,4 \%$ tecnología médica, $1,1 \%$ psicología y $1,2 \%$ otras profesiones (odontología, farmacia y bioquímica, y obstetricia). Entre quienes reportaron estudios técnicos en salud, completos o incompletos, 7,3\% estudiaron enfermería técnica, y $4,7 \%$ otras carreras de salud.

\section{ALGUNAS OMISIONES}

En los registros y encuestas de salud poblacional, tan importante como lo que se indaga, es lo que no se llega a indagar. Existen aspectos sensibles de las experiencias migratorias que no llegan a ser parte de encuestas o cuestionarios por razones de diseño, metodologías, instrumentos, presupuesto, así como por sus propios objetivos, o por las dificultades técnicas para recoger información. Por lo mismo, se necesitarán nuevas investigaciones, que ayuden a comprender la situación, y a organizar mejor las respuestas.

Es el caso, por ejemplo, de la salud mental, considerando que la ENPOVE es una encuesta no especializada, el nivel reportado de «depresión, miedo, enojo, ansiedad, estrés, etc.», fue de $1,9 \%$. Aunque no es metodológicamente comparable, está por debajo de la registrada en adultos en Lima, en quienes en los últimos doce meses la morbilidad mental sentida ha sido estimada en $11,7 \%$ (8\% en hombres, $15,2 \%$ en mujeres) ${ }^{(15)}$, explicable también por la estructura de edades de la población venezolana.

Tampoco se indagó respecto del consumo de alcohol o drogas ilegales, pero si acerca de los «cambios fuertes en los comportamientos o actitudes», durante el mes anterior a la entrevista, que en adolescentes de 12 a 17 años fue reportado en uno de cada cuatro. Otra omisión importante es la salud buco-dental o aspectos nutricionales sobre los que no se incluyeron preguntas. Asimismo, el acceso a servicios en personas con discapacidad tampoco fue evaluado.

\section{DISCUSIÓN}

¿Cuáles son las implicancias de las necesidades y demandas de salud de la población migrante venezolana identificadas en las ciudades del Perú y qué retos suponen para el sistema de salud? En esta sección discutiremos algunas de las implicancias derivadas de la información disponible, sea para la atención en general, como para la salud sexual y reproductiva, según el acceso a servicios y a experiencias de discriminación y violencia. Asimismo, las oportunidades y retos generados por esta inédita experiencia, para la población migrante y local, y para el sistema de salud.

Las condiciones de vida y de salud de la población venezolana en el Perú no debieran verse al margen de las que ya enfrentan las poblaciones urbanas del Perú, sobre todo las más pobres. Tampoco al margen de lo que muestran otras experiencias migratorias que comparten rasgos de vulnerabilidad social comunes, su «relativa desprotección de un grupo de personas cuando enfrentan daños potenciales a su salud, amenazas a la satisfacción de sus necesidades y violación a sus derechos humanos por no contar con recursos personales, sociales y legales» ${ }^{(16)}$.

De particular interés resulta la poca atención a sus necesidades en salud sexual y reproductiva, cuyos indicadores están muy por debajo en el acceso y uso de servicios en referencia a la urbana del Perú. En esas condiciones, resultaría probable que la mayoría de embarazos sigan siendo no planificados, con los riesgos de abortos, y a la larga muertes maternas.

La violencia sexual contra las mujeres inmigrantes venezolanas, que soloestadísticamente consideraríamos semejante al de mujeres peruanas unidas, está lejos de poder ser caracterizada únicamente desde esa perspectiva. En un contexto de insuficiente respuesta social e institucional, las respuestas desde la seguridad ciudadana y de la salud mental requerirían aun mayor atención.

Otro de los hallazgos de la ENPOVE ha sido la llegada de estudiantes, técnicos y profesionales en carreras de salud, lo que ofrecería la posibilidad de atender no sólo a sus compatriotas, sino también a la población peruana. Se facilitaría su integración social, económica y cultural. De superar las dificultades reportadas para homologar sus estudios y títulos, y con los debidos procesos de acreditación, pudieran ser incorporarlos a los servicios. No olvidemos el déficit de profesionales y especialistas, en parte explicado por la emigración de los más de cuarenta mil profesionales de la salud que dejaron el Perú de 1990 al $2017^{(17)}$, aunque no todos de manera definitiva.

Hay asimismo ausencias que requieren mayor atención, a fin de recogerlas en los registros administrativos, o en 
nuevas actualizaciones de la ENPOVE, entre los cuales destacan las condiciones de salud y acceso a servicios de las personas con discapacidad según tipos.

Es claro que este trabajo tiene algunas limitaciones, derivadas en primer lugar de lo que recogen las encuestas utilizadas (condiciones auto-reportadas), así como por no contar con otras fuentes, en particular la que se recoge en los servicios de salud. Asimismo, algunas de las comparaciones de referencia con la población urbana pudieran ser de poca utilidad o hasta forzadas, en razón de sus diferencias metodológicas. Limitaciones también de usar sólo información cuantitativa, que no captura cabalmente percepciones y experiencias de la población de interés.

\section{CONCLUSIONES Y RECOMENDACIONES}

La repentina condición de país de destino migratorio ha planteado múltiples desafíos al sistema de salud en el Perú. Las condiciones de vulnerabilidad social de la población venezolana en las ciudades del Perú revelan y hacen aún más precarias las condiciones de atención de la salud de la población urbana, con la que eventualmente compiten por los mismos insuficientes servicios.

Las necesidades y demandas de atención, promoción y prevención de la salud de la población venezolana están muy influenciadas por el perfil de edades, de interés para organizar y entregar servicios. Los registros administrativos en salud no vienen distinguiendo adecuadamente a la población venezolana.
La situación y determinantes de la salud de la población inmigrante requieren que la gestión de la información, incluyendo los registros administrativos, faciliten las acciones de promoción y prevención adecuadas a su condición y distribución territorial. Para ello, incluso se debiera considerar el rol que profesionales y técnicos calificados para que, de acuerdo a las regulaciones establecidas en el Perú, pudieran sumarse para atender a sus necesidades.

Áreas de mayor prioridad y aún poco atendidas como la salud sexual y reproductiva, y otras insuficientemente caracterizadas como las de violencia física y sexual, desnutrición, discapacidad, así como la salud mental y otras no estimadas como la salud buco-dental, requieren ser visibilizadas, a fin de sustentar intervenciones urgentes para que ningún inmigrante se siga quedando atrás.

Finalmente, la situación analizada ratifica que las desigualdades para ejercer el derecho a la salud, y que también afecta a la población venezolana en nuestro país, continúa siendo uno de los principales retos para que el crecimiento y desarrollo incluyan a quienes afrontan situaciones de mayor vulnerabilidad.

Contribuciones de autoría: WM planteó el plan de análisis y redactó una versión preliminar del artículo. JJM revisó críticamente el contenido e hizo sugerencias sustantivas al mismo. Ambos aprobaron la versión final.

\section{Fuentes de financiamiento: Autofinanciado.}

Conflictos de interés: Junto a otras agencias internacionales (ACNUR, Banco Mundial, OIM, UNICEF), WM coordinó la asistencia técnica y financiera que UNFPA brindó al INEI para la realización de la ENPOVE. JJM no declara conflictos de interés.

\section{REFERENCIAS BIBLIOGRÁFICAS}

1. Instituto Nacional de Estadística e Informática; Perú: Estadísticas de la Emigración Internacional de peruanos e Inmigración de Extranjeros, 19902017. Lima: 2018.

2. Grupo de Trabajo de Refugiados y Migración. Lima; 2019 Peru - National Coordination Platform for Migrants and Refugees - Situation Report - June 2019 [Internet]. [citado el 22 de julio 2019]. (Disponible en: https://r4v.info/es/ documents/download/70388).

3. Consejo de Derechos Humanos. Informe de la Alta Comisionada de las Naciones Unidas para los Derechos Humanos sobre la situación de los derechos humanos en la República Bolivariana de Venezuela, $41^{\circ}$ período de sesiones, 24 de junio- 12 de julio de 2019 [Internet]. Geneva: Office of the United Nations High Commissiones for Human Rights; 2019 [citado el 13 de julio 2019]. (Disponible en: https:// www.ohchr.org/EN/HRBodies/ HRC/RegularSessions/Session41/ Documents/A_HRC_41_18_SP.docx,).

4. Roa AC. Sistema de salud en Venezuela: ¿un paciente sin remedio? Cad Saúde Pública. 2018;34(3):e00058517. doi: 10.1590/0102-311X00058517.

5. Contraloría General de la República. Operativo de Control. "Por una Salud de Calidad" del 28 de mayo al 1 de junio 2018. Lima, Perú: CGR;2018.

6. Instituto Nacional de Estadística e Informática. Características sociodemográficas de la población venezolana censada en el año 2017. Lima, Perú:INEI;2018.

7. Instituto Nacional de Estadística e Informática. Condiciones de Vida de la Población Venezolana que reside en Perú. "Resultados de la Encuesta dirigida a la Población Venezolana que reside en el Perú" ENPOVE 2018. Lima, , Perú:INEI;2019.

8. Organización Internacional para las Migraciones (OIM). Monitoreo de Flujo de la Población Venezolana en el Perú DTM Ronda 3 [Internet]. Lima, Perú: OIM; 2018. [citado el 15 de abril 2019]. (Disponible en: https://reliefweb. int/sites/reliefweb.int/files/resources/ DTM_R3_FlowMonitoring_OIMPeru. pdf).

9. Instituto Nacional de Estadística e Informática. Perú: Estimaciones y Proyecciones de la Población Nacional, por Años Calendario y Edades Simples, 1950-2050, Boletín Especial № 24, Lima, Perú: INEI; 2019. 
10. AbubakarI,Aldridge RW,DevakumarD, Orcutt $\mathrm{M}$ et al. The UCL-Lancet Commission on Migration and Health: the health of a world on the move. Lancet. 2018;392(10164):2606-2654. doi: 10.1016/S0140-6736(18)32114-7.

11. Instituto Nacional de Estadística e Informática. Condiciones de Vida en el Perú. Trimestre: Octubre-NoviembreDiciembre 2018. Informe Técnico $N^{\circ} 1$, marzo 2019. Lima, Perú: INEI, 2019.

12. Instituto Nacional de Estadística e Informática. Condiciones de Vida en el Perú. Trimestre: Enero-Febrero-Marzo 2019. Informe Técnico No2, junio 2019. Lima, Perú: INEI, 2019.
13. Rebolledo-Ponietsky, KA, Munayco CV, Mezones-Holguín E. Migration crisis in Venezuela: impact on HIV in Peru. J Travel Med. 2019;26(2). pii: tay155. doi: 10.1093/jtm/tay155.

14. Silva Santisteban A. Diagnóstico rápido: Situación de los migrantes venezolanos con VIH en el Perú. Lima: ONUSIDA; 2019.

15. Instituto Nacional de Salud Mental (Perú). Estudio Epidemiológico de Salud Mental en Lima Metropolitana y Callao Replicación 2012. Informe General. Anales de Salud Mental. 2013;XXIX, Suplemento 1.

16. Salgado de Snyder VN, González Vázquez T, Bojorquez Chapela L, Infante
Xibille C. Vulnerabilidad social, salud y migración México-Estados Unidos. Salud Pública Méx. 2007;49(sup 1):8-10.

17. Instituto Nacional de Estadística e Informática, Superintendencia de Migraciones, OIM, Ministerio de RR.EE. Perú: Estadísticas de la Emigración Internacional de peruanos e Inmigración de Extranjeros, 1990 - 2017. Lima, Perú: INEI, 2019.

Correspondencia: Walter Mendoza

Correoelectrónico:mendoza@unfpa.org

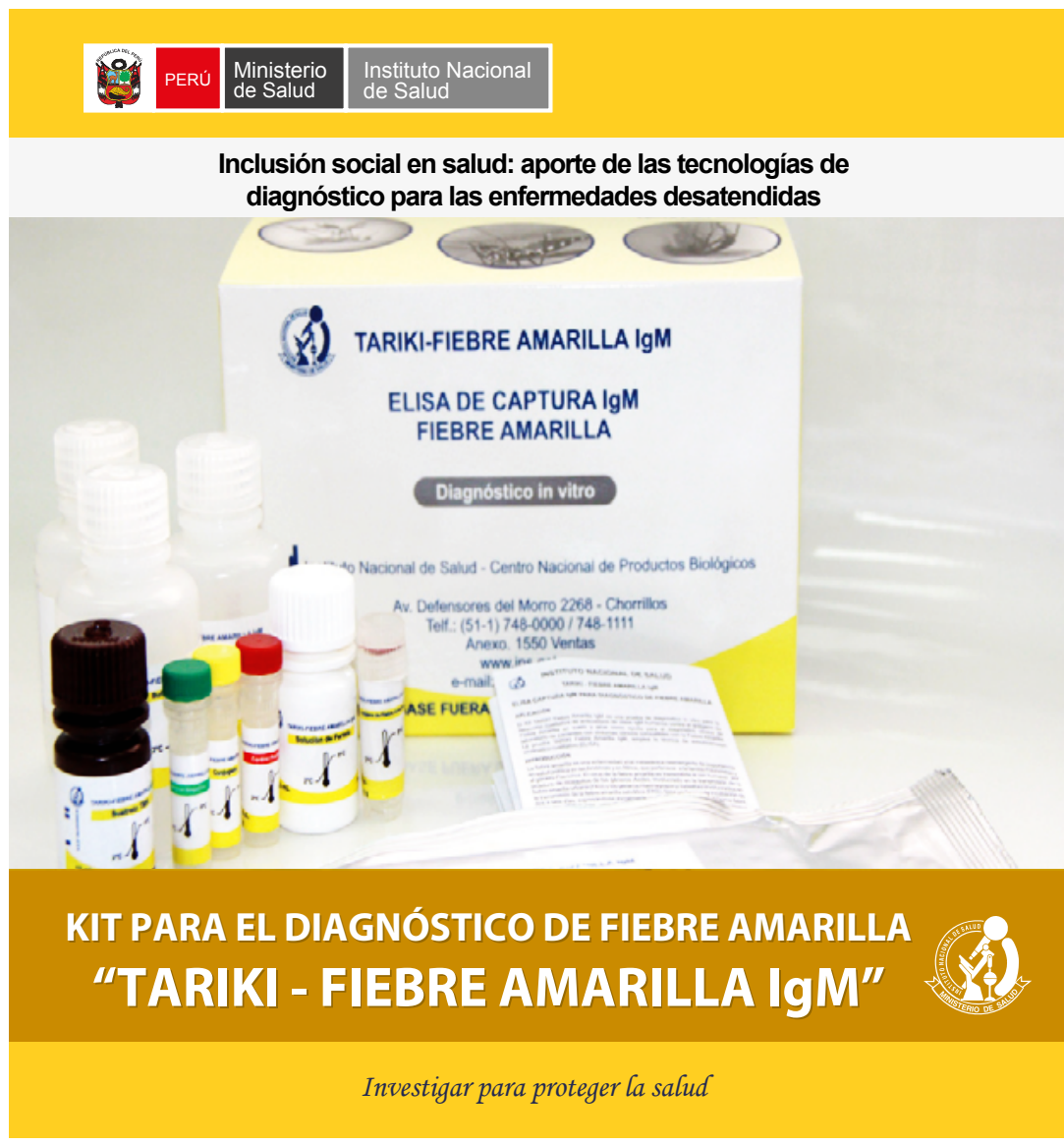

